\section{References}

1. Cheng VC, Wong SC, Chan VW, et al. Air and environmental sampling for SARS-CoV-2 around hospitalized patients with coronavirus disease 2019 (COVID-19). Infect Control Hosp Epidemiol 2020;41:1258-1265.

2. Chia PY, Coleman KK, Tan YK, et al. Detection of air and surface contamination by SARS-CoV-2 in hospital rooms of infected patients. Nat Commun 2020;11:2800.

3. Guo ZD, Wang ZY, Zhang SF, et al. Aerosol and surface distribution of severe acute respiratory syndrome coronavirus 2 in hospital wards, Wuhan, China, 2020. Emerg Infect Dis 2020;26:1583-1591.

4. Jerry J, O’Regan E, O'Sullivan L, Lynch M, Brady D. Do established infection prevention and control measures prevent spread of SARS-CoV-2 to the hospital environment beyond the patient room? J Hosp Infect 2020;105:589-592.

5. Lei $\mathrm{H}, \mathrm{Ye} \mathrm{F}$, Liu X, et al. SARS-CoV-2 environmental contamination associated with persistently infected COVID-19 patients. Influenza Other Respir Viruses 2020;14:688-699.

6. Li YH, Fan YZ, Jiang L, Wang HB. Aerosol and environmental surface monitoring for SARS-CoV-2 RNA in a designated hospital for severe COVID-19 patients. Epidemiol Infect 2020;148:e154.

7. Ong SWX, Tan YK, Chia PY, et al. Air, surface environmental, and personal protective equipment contamination by severe acute respiratory syndrome coronavirus 2 (SARS-CoV-2) from a symptomatic patient. JAMA 2020; 323:1610-1612.
8. Razzini K, Castrica M, Menchetti L, et al. SARS-CoV-2 RNA detection in the air and on surfaces in the COVID-19 ward of a hospital in Milan, Italy. Sci Total Environ 2020;742:140540.

9. Santarpia JL, Rivera DN, Herrera VL, et al. Aerosol and surface contamination of SARS-CoV-2 observed in quarantine and isolation care. Sci Rep 2020;10:12732.

10. Wei L, Lin J, Duan X, et al. Asymptomatic COVID-19 patients can contaminate their surroundings: an environment sampling study. mSphere 2020;5: e00442-20.

11. Zhou J, Otter JA, Price JR, et al. Investigating SARS-CoV-2 surface and air contamination in an acute healthcare setting during the peak of the COVID-19 pandemic in London. Clin Infect Dis 2020. doi: 10.1093/cid/ ciaa905.

12. Kanamori H, Weber DJ, Rutala WA. Role of the healthcare surface environment in SARS-CoV-2 transmission and potential control measures. Clin Infect Dis 2021;72:2052-2061. doi: 10.1093/cid/ciaal467.

13. Kanamori H, Rutala WA, Sickbert-Bennett EE, Weber DJ. Review of fungal outbreaks and infection prevention in healthcare settings during construction and renovation. Clin Infect Dis 2015;61:433-444.

14. Morawska L, Milton DK. It is time to address airborne transmission of COVID-19. Clin Infect Dis 2020;71:2311-2313.

15. Tang S, Mao Y, Jones RM, et al. Aerosol transmission of SARS-CoV-2? Evidence, prevention and control. Environ Int 2020;144:106039.

\title{
Big data-driven personal protective equipment stockpiling framework under a universal healthcare system for disease control and prevention in the COVID-19 era
}

\author{
Kevin Sheng-Kai Ma DDS, MSc (1) and Alice Shin-Yi Tsai MD, MS, DrPH \\ Department of Health Policy and Management, Johns Hopkins University Bloomberg School of Public Health, Baltimore, Maryland
}

To the Editor-We appreciate the letter by Wang et $\mathrm{al}^{1}$ regarding the issue of face masks in protecting against the coronavirus disease 2019 (COVID-19) outbreak. In this letter, we report the distribution of surgical masks on a real-time basis and recognition of the mask holders in need. We hope that by introducing this system to increase the distribution channels, the burden on healthcare providers can be reduced and that the user-friendly interface for PPE providers and consumers may help expedite PPE distribution in a more efficient manner.

Big data analytics has improved healthcare by analyzing electronic medical records, sociodemographic information, and environmental factors. ${ }^{2}$ Moreover, its tracking roles in emerging infectious diseases, including the coronavirus pandemic, have been discussed. ${ }^{3}$ In countries with single-payer universal healthcare systems (UHSs), claimed data of payers could be an abundant source for analytics. On the other hand, compulsory social distancing, coupled with mass masking, has been widely adopted as a strategy for nonspecific symptoms in early-stage COVID- $19 .{ }^{4}$ We propose

Author for correspondence: Kevin Sheng-Kai Ma, E-mail; Kevinskma1@gmail.com Cite this article: Ma KS-K and Tsai AS-Y. (2022). Big data-driven personal protective equipment stockpiling framework under a universal healthcare system for disease control and prevention in the COVID-19 era. Infection Control \& Hospital Epidemiology, 43: 133-134, https://doi.org/10.1017/ice.2020.387 that analytics based on proper concatenation of databases may prevent supply shortages of personal protective equipment (PPE).

Taking Taiwan as an example, cloud-computing-based healthcare databases within the UHS has alleviated the integration between primary care providers and hospitals and has reduced the cost of tracking. Applying the same logistics to PPE allocation would allow PPE providers to manage the distribution of surgical masks on a real-time basis and to recognize the mask holders according to insurance or passport number. ${ }^{5}$ With the help of data analysis, combining artificial intelligence and cloud technology, public health policy making could be practicable. Thus, when it comes to the implementation cost of epidemic prevention policies, Taiwan authorities adopt low-cost, stringent-level strategies compared with other high-income countries, but they still achieved epidemic control in the early outbreak. ${ }^{6}$

After the 2003 severe acute respiratory syndrome (SARS) outbreak, the Taiwan CDC (TCDC) started transferring registered real-time infectious disease data to this established monitoring system, in which PPE stockpiling platform was used. Therefore, prior to the official recognition of COVID-19 outbreak, ${ }^{3}$ PPE databases were subsequently concatenated by UHS to manage resource allocation and logistics when several cases were identified. Establishment of this application programming interface for mask-selling pharmacies under UHS required data transfers as well as managerial

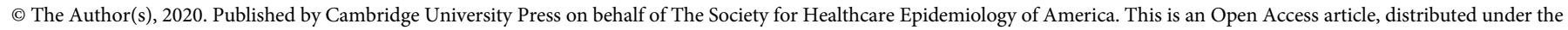
terms of the Creative Commons Attribution licence (http://creativecommons.org/licenses/by/4.0/), which permits unrestricted re-use, distribution, and reproduction in any medium, provided the original work is properly cited. 
issues including governance and ownership, for which interdepartmental communication was efficient within the UHS. Specifically, the tracking system expanded the healthcare informatics system that pharmacists were familiar with, and its user-friendly interfaces for PPE providers and consumers helped expedite distribution processes. $^{5}$ The UHS and the TCDC have also promoted the system to increase the distribution channels, within which government offices may also allot masks to lessen the burden on healthcare providers.

Because masks alone are not effective without combining infection-control measures, ${ }^{7}$ we recommend this integrative platform for the maintenance of more PPE stockpiles, including critical infection-control equipment to reduce iatrogenic SARS-CoV-2 exposure.

\section{Acknowledgments.}

Financial support. No financial support was provided relevant to this article. article.

\section{References}

1. Wang MW, Cheng YR, Ye L, Zhou MY. The COVID-19 outbreak: the issue of face masks. Infect Control Hosp Epidemiol 2020 April 14 [Epub ahead of print]. doi: 10.1017/ice.2020.129.

2. Vigilante K, Escaravage S, McConnell M. big data and the intelligence community-lessons for health care. N Engl J Med 2019;380:1888-1890.

3. Wang CJ, Ng CY, Brook RH. Response to COVID-19 in Taiwan: big data analytics, new technology, and proactive testing. JAMA 2020;323:1341-1342.

4. Ranney ML, Griffeth V, Jha AK. Critical supply shortages- the need for ventilators and personal protective equipment during the COVID-19 pandemic. N Engl J Med 2020;382:e41. doi: 10.1056/NEJMp2006141.

5. National Health Command Center. Taiwan Centers for Disease Control website. https://www.cdc.gov.tw/En/Category/MPage/gL7-bARtHyNdrDq882pJ9Q. Updated February 1, 2018. Accessed May 6, 2020.

6. Hale T, Webster S, Petherick A, Phillips T, Kira B. Oxford COVID-19 government response tracker. Blavatnik School of Government. Data use policy: Creative Commons Attribution CC BY standard. 2020.

7. Klompas M, Morris CA, Sinclair J, Pearson M, Shenoy ES. Universal masking in hospitals in the COVID-19 era. NEngl J Med 2020. doi: 10.1056/NEJMp2006372.

\title{
Impact of early carbapenemase notification on infection control management and antimicrobial stewardship
}

\author{
Leandro Reus Rodrigues Perez $\mathrm{PhD}^{1}$ (1), Gabriel Narvaez $\mathrm{MD}^{2}$ and Cícero Dias PhD \\ ${ }^{1}$ Federal University of Health Sciences of Porto Alegre, Porto Alegre, Brazil and ${ }^{2}$ Mãe de Deus Hospital, Porto Alegre, Brazil
}

To the Editor-The worldwide spreading of carbapenemaseproducing Enterobacterales (CPE) is a matter of concern due to the limited therapeutic options available. ${ }^{1}$ In severe cases of infection, an early carbapenemase detection and notification is crucially important for the adequacy of antimicrobial treatment, for the management of patients, and to establish infection control practices. $^{2}$ Some microbiology laboratories have used blue-carba, a colorimetric test, because it is fast, easy to read, and inexpensive. ${ }^{3}$ However, the impact for the infection control practices and the turnaround time of its use on previous carbapenemase detection among Enterobacterales has been poorly evaluated.

We aimed to determine the turnaround time until CPE notification in comparison with the time to report a final microbiology result (bacterial identification plus antimicrobial susceptibility testing). We also aimed to evaluate the importance of this notification for the infection control measures and antimicrobial resistance predictability.

During a follow-up survey from August 2017 to August 2018, we performed an observational study in patients at a tertiary-care hospital from Porto Alegre, Brazil. Enterobacterales isolates

Author for correspondence: Leandro Reus Rodrigues Perez, E-mail: leandro.reus@ gmail.com

PREVIOUS PRESENTATION. This information was presented in part as abstract no. ECCMD-5455 in the "Better together: antibiotic stewardship and infection control" session of the $30^{\text {th }}$ European Congress of Clinical Microbiology and Infectious Diseases in Paris, France, on April 21, 2020.

Cite this article: Perez LRR, Narvaez G, and Dias C. (2022). Impact of early carbapenemase notification on infection control management and antimicrobial stewardship. Infection Control \& Hospital Epidemiology, 43: 134-136, https://doi.org/ 10.1017/ice.2020.1391 recovered from any clinical specimen were submitted to blue-carba test (BCT) for phenotypic carbapenemase detection. ${ }^{3}$ Isolates were identified using Vitek 2 (bioMérieux, Marcy l'Etoile, France) or MALDI-TOF/MS (Bruker Daltonics, Germany), if necessary. Antimicrobial susceptibility testing was determined by disc diffusion (Oxoid, for amikacin, gentamycin, meropenem; Etest (bioMérieux, Marc l'Étoile, France) for fosfomycin (when isolates were recovered from urine) and broth microdilution for polymyxin $\mathrm{B}$ and tigecycline. Carbapenemase characterization was conducted using phenotypic tests using specific inhibitors, as described elsewhere. 4

The work flow required the microbiology laboratory to notify the infection control staff or clinician of a positive BCT result for early carbapenemase notification after bacterial isolation from each clinical specimen analyzed.

During the period of the study, 300 CPE notifications were made, including 155 distinct patients. The average time was 1.19 days for CPE notification versus 2.38 days for the final report (Fig. 1). KPC-producing Klebsiella pneumoniae was the most prevalent agent (291 of $300,97 \%$ ) and no other gene carbapenemase than $b l a_{\mathrm{KPC}-2}$ was detected during this period. Antimicrobial resistance was observed as follows: meropenem 97.7\%, gentamicin 77.6\%, fosfomycin 31.6\%, polymyxin B $29.0 \%$, amikacin $7.3 \%$ and tigecycline $5 \%$.

Of the 155 patients enrolled in this survey, in 73 patients (47.1\%) an adjustment of antimicrobial therapy was promoted after the early BCT notification. These adjustments were due to the inclusion of polymyxin B $(65.7 \%, 48$ patients), amikacin (28.8\%, 21 patients), or fosfomycin (5.5\%, 4 patients). For 25 patients $(16.1 \%)$, no change in initial therapy was verified. In these 